\title{
Reacreditación del Programa de Enfermería de la Universidad Francisco de Paula Santander: Trabajando por la calidad de la educación y el cuidado.
}

\author{
Dianne Sofia González-Escobar*
}

En la Universidad Francisco de Paula Santander el concepto de autoevaluación se encuentra enunciado en el Estatuto General (1), como la "Evaluación permanente entendida como método y práctica en todos los niveles de la institución y como actitud hacia la autocrítica y la revisión de resultados, bases para las decisiones de mejoramiento". Bajo este referente y atendiendo las políticas nacionales de calidad de la educación superior, en el año 2002 se adopta el proceso de autoevaluación institucional como "política de máxima prioridad y cumplimiento por parte de los órganos de dirección y gobierno de la universidad y sus dependencias de apoyo en orden académico, administrativo y de bienestar de la universidad, hacia el mejoramiento institucional" (2).

A partir de ese momento, en la institución se generan procesos de reflexión y análisis que concluyen en la construcción de lineamientos, políticas y modelos que fortalecen el desarrollo de las funciones misionales, así como de los procesos académicos y administrativos. En este ejercicio se define el Modelo de Autoevaluación, el cual adopta los lineamientos planteados por el Consejo Nacional de Acreditación (CNA) y que, en su implementación, busca la construcción de una cultura de calidad y de mejoramiento continuo.

El Programa de Enfermería, considerando el logro de altos estándares de calidad en el marco de la normativa institucional y del sector educativo y de salud, por su naturaleza, es pionero en la Universidad por realizar el ejercicio de autoevaluación con fines de acreditación, con el apoyo del equipo asesor de autoevaluación y acreditación institucional y la participación activa de la comunidad académica integrada por estudiantes, docentes, egresados y administrativos, así como el sector productivo y la comunidad en general. Producto de este ejercicio se obtiene la acreditación de alta calidad del Programa, otorgada por el Ministerio de Educación Nacional, según Resolución N 4790 del 8 de mayo de 2012.
El proceso de acreditación creó en el programa una dinámica que consolidó la cultura de la calidad, lo cual fortaleció el análisis permanente para la construcción y deconstrucción de diferentes componentes del plan de estudios, así como el desarrollo de las acciones de mejoramiento y mantenimiento planteadas como producto de la autoevaluación. Por ello, el trabajo para la reacreditación inició en el primer semestre de 2013, centrado tanto en la implementación del plan de mejoramiento como en el cumplimiento de los atributos de calidad definidos en los lineamientos para la acreditación de programas de pregrado del CNA (2013), que contempla los factores, las características y los aspectos a evaluar.

El proceso se desarrolló a través de las diferentes etapas definidas por el modelo de autoevaluación institucional: sensibilización, ponderación, recolección de información, análisis de información, emisión de juicios, definición del plan de mejoramiento y socialización de resultados, bajo un enfoque de análisis descriptivo, identificando inicialmente el alcance de cada característica a evaluar, especialmente aquellas nuevas definidas por el CNA en el documento del año 2013. El liderazgo y coordinación del proceso estuvo a cargo de la directora del programa y el Comité Curricular, con el grupo de trabajo de autoevaluación del programa constituido por el colectivo docente de tiempo completo. Metodológicamente, se definieron grupos de trabajo por factor, de acuerdo con la afinidad de los docentes frente a cada uno de ellos. En cada una de las etapas se contó con el acompañamiento del Comité de Autoevaluación Institucional.

De los resultados del proceso de autoevaluación del programa 2013 - 2015, se destaca (3):

- La apropiación de los elementos estratégicos por parte de la comunidad académica y la pertinencia del Proyecto Educativo
ISSN-PRINT

1794-9831

E-ISSN 2322-7028

Vol. 14 No. 2

Jul - Dic 2017

Cúcuta, Colombia

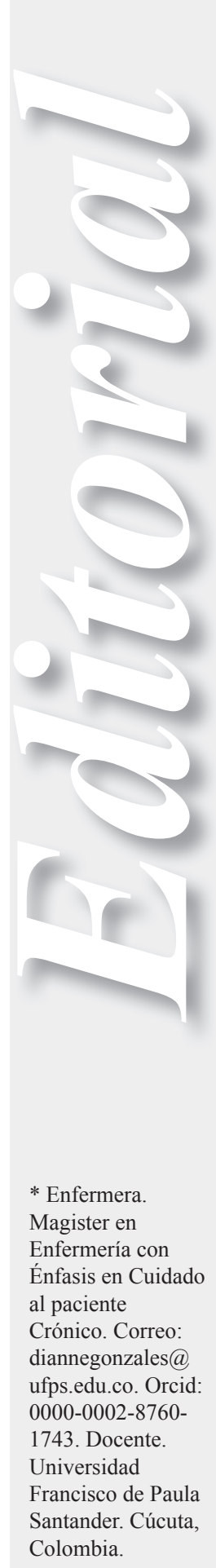


1794-9831

E-ISSN 2322-7028

Vol. 14 No. 2

Jul - Dic 2017

Cúcuta, Colombia
Institucional y del Programa, evidenciado en el reconocimiento que existe de estos elementos en la comunidad, aspecto que permite el desarrollo de la funciones sustantivas y los elementos que constituyen el quehacer de la universidad y del programa en el contexto local, regional y binacional. Para Enfermería, la formación de profesionales líderes, comprometidos y competentes frente a los retos que el medio les plantea, es quizá una de las más grandes apuestas que se desarrollan en aras del cumplimiento de su misión.

- $\quad$ El Programa cuenta con convenios de Relación Docencia Servicio que muestran la equidad y pertinencia según la normatividad a nivel nacional, con instituciones habilitadas y en proceso de solicitar la acreditación, lo cual permite a los estudiantes desarrollar las competencias según el nivel de formación y complejidad.

- $\quad$ El desempeño y los resultados en las pruebas Saber PRO 2013 - 2014 fueron superiores al promedio nacional en las áreas de lectura crítica, razonamiento cuantitativo, competencias ciudadanas y comunicación escrita.

La Institución y el Programa de Enfermería ofrecen garantías y demuestran equidad en el apoyo económico destinado para la capacitación formal y no formal del profesorado. El Programa cuenta con docentes de alta calidad profesional, teniendo en cuenta que se promueve la formación y capacitación en áreas disciplinares y básicas para el mejoramiento docente cuyo impacto se refleja en una mejor utilización de la tecnología y el incremento de actividades investigativas.
El Programa de Enfermería tiene una estructura curricular orientada a la integralidad y la flexibilidad que permite y promueve la movilidad, el diseño del currículo por competencias y la aplicación de estrategias diversas de enseñanza-aprendizaje que promueven el modelo dialógico crítico, además de la integración de nuevas estrategias como cursos de inglés aunados a los cursos para el dominio de una segunda lengua. Los sistemas de evaluación de los estudiantes son transparentes y coherentes con los contenidos y metodologías de enseñanza, alineados con los principios de coherencia, equidad e integridad.

- El Proyecto Educativo Institucional y del Programa de Enfermería plantean la proyección social como una de sus funciones centrales. El Programa responde a este aspecto misional con la búsqueda de soluciones a los problemas de salud del contexto en que se desarrolla, a través del diseño y ejecución de acciones de promoción de la salud, prevención de la enfermedad, atención y rehabilitación en salud a personas, familias y colectivos en situación de vulnerabilidad. Los resultados obtenidos le han merecido el reconocimiento de la población beneficiaria, instituciones de salud públicas y privadas, así como de organismos gubernamentales del orden regional y nacional.

- El Programa de Enfermería, en los últimos cinco años, ha fortalecido la interacción con comunidades académicas de orden nacional e internacional mediante convenios de movilidad, participación de los docentes en redes $\mathrm{y} / \mathrm{o}$ asociaciones, vinculación a proyectos de investigación tanto de docentes como estudiantes y visita de expertos nacionales e internacionales, entre otros aspectos.

La organización periódica de diferentes actividades académicas, derivadas de las líneas de investigación de los grupos de investigación, ponen en contacto al estudiante con la investigación que se está realizando a nivel local, nacional e internacional y con las tendencias de la investigación y la disciplina.

- $\quad$ El sistema de investigación de la universidad posiciona a la investigación como una función sustancial de la universidad, reconociendo el tiempo dedicado por el docente a la investigación, aumentando los incentivos para los grupos de investigación y los docentes investigadores, incluidos los profesores con vinculación de cátedra.

Las políticas, programas y servicios ofertados reconocen el valor de la diversidad, promueven el respeto por la diferencia, generan ayudas para contrarrestar las situaciones de vulnerabilidad e incentivan con estímulos académicos, económicos y socio-afectivos la permanencia y graduación de sus estudiantes, 
lo cual guarda relación con el principio de eficiencia e idoneidad al hacer uso adecuado de los medios que dispone la institución para lograr el cumplimiento de los propósitos que están enunciados en el PEI

- La existencia de una reglamentación pertinente con las necesidades organizacionales y administrativas de directivos, docentes y estudiantes permite orientar los procesos de toma de decisiones de manera asertiva, adecuada y sustentada, según los lineamientos de la institución.

- La apreciación de los egresados, de los empleadores y de los usuarios externos sobre la calidad de la formación del programa es buena, puesto que consideran que ha favorecido proyectos de vida a nivel personal, profesional y laboral.
El Programa de Enfermería dispone de recursos físicos adecuados y suficientemente dotados para el desarrollo de sus actividades sustantivas y el bienestar de su comunidad académica.

El informe de autoevaluación con fines de reacreditación del Programa de Enfermería permitió demostrar el logro de niveles de calidad suficientes para obtener el reconocimiento público por parte del Ministerio de Educación Nacional mediante Resolución 00184 del 16 de enero de 2017 (4). La renovación de la acreditación confirma el compromiso institucional y del programa para alcanzar la excelencia académica en todos sus procesos, además del desarrollo de sus funciones misionales y el deseo de continuar contribuyendo al desarrollo de la disciplina y profesión de enfermería en la región y el país.

\section{Referencias Bibliográficas}

1. Universidad Francisco de Paula Santander. Acuerdo 091 de 1993, literal F del artículo 4, diciembre 1, por el cual se establece el Estatuto General de la Universidad Francisco de Paula Santander. Cúcuta, 1993.

2. Universidad Francisco de Paula Santander. Consejo Superior Universitario. Acuerdo No. 017 de 15 de marzo de 2002. Política de Autoevaluación Institucional.

3. Universidad Francisco de Paula Santander. Facultad Ciencias de la Salud. Programa de Enfermería. Documento Informe final de autoevaluación con fines de Reacreditación del programa de Enfermería, octubre de 2015.

4. República de Colombia, Ministerio Educación Nacional. Resolución $\mathrm{N}^{\circ} 00184$, enero 16, por medio del cual se otorga la renovación de la acreditación de alta calidad al Programa de Enfermería de la Universidad Francisco de Paula Santander. Bogotá: 2017. 